J. Korean Math. Soc. 50 (2013), No. 5, pp. 991-1008

http://dx.doi.org/10.4134/JKMS.2013.50.5.991

\title{
ON OVERRINGS OF GORENSTEIN DEDEKIND DOMAINS
}

\author{
Kui Hu, Fanggui Wang, Longyu Xu, and Songquan Zhao
}

Abstract. In this paper, we mainly discuss Gorenstein Dedekind domains (G-Dedekind domains for short) and their overrings. Let $R$ be a one-dimensional Noetherian domain with quotient field $K$ and integral closure $T$. Then it is proved that $R$ is a G-Dedekind domain if and only if for any prime ideal $P$ of $R$ which contains $\left(R:_{K} T\right), P$ is Gorenstein projective. We also give not only an example to show that G-Dedekind domains are not necessarily Noetherian Warfield domains, but also a definition for a special kind of domain: a 2-DVR. As an application, we prove that a Noetherian domain $R$ is a Warfield domain if and only if for any maximal ideal $M$ of $R, R_{M}$ is a 2 -DVR.

\section{Introduction}

Throughout this paper, all rings are commutative with identity element and all modules are unitary. Let $R$ be a domain with quotient field $K$ and $J$ a fractional ideal of $R$. The definition of $J^{-1}$ can be found in [14] as follows:

$$
J^{-1}=\{x \mid x \in K, x J \subset R\} .
$$

The definition of divisorial ideals can be found in [10]: A fractional ideal $J$ of $R$ is said to be divisorial if $J_{v}=\left(J^{-1}\right)^{-1}=J$.

Let $R$ be a commutative ring. An $R$-module $M$ is said to be Gorenstein projective ( $G$-projective for short) if there exists an exact sequence of projective modules

$$
\mathbf{P}=\cdots \rightarrow P_{1} \rightarrow P_{0} \rightarrow P^{0} \rightarrow P^{1} \rightarrow \cdots
$$

such that $M \cong \operatorname{Im}\left(P_{0} \rightarrow P^{0}\right)$ and such that $\operatorname{Hom}_{R}(-, Q)$ leaves the sequence $\mathbf{P}$ exact whenever $Q$ is a projective $R$-module. An $R$-module $M$ is said to be strongly Gorenstein projective (SG-projective for short) if there exists an exact sequence of projective modules

$$
\mathbf{P}=\cdots \rightarrow P \rightarrow P \rightarrow P \rightarrow P \rightarrow \cdots
$$

Received September 27, 2012; Revised May 30, 2013.

2010 Mathematics Subject Classification. 13G05, 13D03.

Key words and phrases. Gorenstein projective module, Gorenstein Dedekind domain, strongly Gorenstein projective module, $n$-strongly Gorenstein projective module, Noetherian Warfield domain, 2-DVR. 
such that all these projective modules and all the morphisms of the exact sequence are the same and $M \cong \operatorname{Im}(P \rightarrow P)$ and also, $\operatorname{Hom}_{R}(-, Q)$ leaves the sequence $\mathbf{P}$ exact whenever $Q$ is a projective $R$-module.

The concepts of G-hereditary rings and G-Dedekind domains were introduced in [16]. It can be seen from [12, Theorem 2.28] and [20, Theorem 6.3.4] that a one-dimensional Noetherian domain either is a G-Dedekind domain or has infinite Gorenstein global dimension. Let $R$ be a one-dimensional Noetherian domain with quotient field $K$ and integral closure $T$. It is proved in the first section that $R$ is a G-Dedekind domain if and only if for any prime ideal $P$ of $R$ which contains $\left(R:_{K} T\right), P$ is G-projective.

An overring of a domain $R$ is a ring between $R$ and its quotient field $K$. It is well known that any overring of a Dedekind domain is still a Dedekind domain. It is natural to ask whether overrings of a G-Dedekind domain are GDedekind domains or not? In Section 2, we give an example which shows that this is not necessarily the case. In fact, it can be seen from [19, Theorem 6.3] and [19, Theorem 6.4] that a Noetherian domain has the property that each of its overring is a G-Dedekind domain if and only if it is a Warfield domain. In [5] and [6], Driss Bennis and Najib Mahdou introduced strongly Gorenstein projective modules and $n$-strongly Gorenstein projective modules respectively. In Section 2, we utilize these concepts as tools to investigate some properties of Noetherian Warfield domains.

The author in [19] gave a general introduction to Warfield domains. A domain $R$ is called a Warfield domain if, given any submodule $A$ of the field of quotients $K$, the $A$-torsionless $\operatorname{End}_{R}(A)$-modules of finite rank are $A$-reflexive. In Section 2, we not only provide an example to show that G-Dedekind domains are not necessarily Noetherian Warfield domains, but also give a definition for a special kind of domain: a 2-DVR. A Noetherian local domain is called a 2-DVR if its maximal ideal is strongly G-projective. As an application, we prove that a Noetherian domain $R$ is a Warfield domain if and only if for any maximal ideal $M$ of $R, R_{M}$ is a 2 -DVR.

\section{One-dimensional Noetherian domains and Gorenstein Dedekind domains}

We say that a module $M$ has Gorenstein projective dimension at most a positive integer $n$, and we write $G p d(M) \leqslant n$, if there exists an exact sequence of modules

$$
0 \rightarrow G_{n} \rightarrow \cdots \rightarrow G_{0} \rightarrow M \rightarrow 0
$$

where each $G_{i}$ is G-projective.

A module $M$ is said to be Gorenstein injective, if there exists an exact sequence of injective modules

$$
\mathbf{I}=\cdots \rightarrow I_{1} \rightarrow I_{0} \rightarrow I^{0} \rightarrow I^{1} \rightarrow \cdots
$$


such that $M \cong \operatorname{Im}\left(I_{0} \rightarrow I^{0}\right)$ and such that $\operatorname{Hom}_{R}(E,-)$ leaves the sequence $\mathbf{I}$ exact whenever $E$ is an injective module.

We say that a module $M$ has Gorenstein injective dimension at most a positive integer $n$ and we write $\operatorname{Gid}(M) \leqslant n$, if there exists an exact sequence of modules

$$
0 \rightarrow M \rightarrow G_{0} \rightarrow \cdots \rightarrow G_{n} \rightarrow 0,
$$

and each $G_{i}$ is Gorenstein injective. The authors in [7] have studied global Gorenstein dimensions of a ring $R$, which are called Gorenstein projective, injective, and weak dimensions of $R$, denoted by $\operatorname{GPD}(R), \operatorname{GID}(R)$, and $\mathrm{G}-w \operatorname{dim}(R)$, respectively, defined as follows:

$$
\begin{aligned}
\operatorname{GPD}(R) & =\sup \{G p d(M) \mid M R \text {-module }\} \\
\operatorname{GID}(R) & =\sup \{G i d(M) \mid M R \text {-module }\}, \text { and } \\
\mathrm{G}-w \operatorname{dim}(R) & =\sup \left\{G f d_{R}(M) \mid M R \text {-module }\right\} .
\end{aligned}
$$

They proved that for any $\operatorname{ring} R, \operatorname{G}-w \operatorname{dim}(R) \leqslant \operatorname{GID}(R)=\operatorname{GPD}(R)[7$, Theorems 3.2 and 4.2]. The common value of $\operatorname{GPD}(R)$ and $\operatorname{GID}(R)$ is called Gorenstein global dimension of $R$, and denoted by $G-g l \operatorname{dim}(R)$.

If $R$ is a ring of finite global dimension (denoted by $g l \cdot \operatorname{dim}(R)<\infty$ ), then every Gorenstein projective module over $R$ is projective since its projective dimension is finite. Therefore $g l \cdot \operatorname{dim}(R)=G-g l \operatorname{dim}(R)$.

A domain is called a Gorenstein Dedekind domain (G-Dedekind domain for short) if every submodule of a projective module is G-projective (i.e., $G-g l \operatorname{dim}(R) \leqslant 1)$. Dedekind domains are G-Dedekind domains since their Gorenstein global dimensions are equal to their global dimensions and must be at most 1 .

Recall that the FPD dimension of a ring $R$ is defined as follows:

$\operatorname{FPD}(R)=\sup \left\{p d_{R}(M) \mid M\right.$ is an $R$-module such that $\left.p d_{R}(M)<\infty\right\}$, where $p d_{R}(M)$ denotes projective dimension of $M$. Likewise in [12], the FGPD dimension of a ring $R$ is defined as follows:

$\operatorname{FGPD}(R)=\sup \left\{\operatorname{Gpd}_{R}(M) \mid M\right.$ is an $R$-module such that $\left.\operatorname{Gpd}_{R}(M)<\infty\right\}$.

The following theorem is [12, Theorem 2.28].

Theorem 1.1. For any ring $R$ there is an equality: $\operatorname{FGPD}(R)=\operatorname{FPD}(R)$.

For the relation between $\operatorname{FPD}(R)$ and Krull dimension $\operatorname{dim}(R)$, we have the following theorem [20, Theorem 6.3.4] (its proof can be seen in [11]):

Theorem 1.2. For any Noetherian ring $R$ there is an equality: $\operatorname{FPD}(R)=$ $\operatorname{dim}(R)$.

Therefore, for any Noetherian ring $R$, we surely have $\operatorname{FGPD}(R)=\operatorname{dim}(R)$. If $R$ is a one-dimensional Noetherian domain (i.e., $\operatorname{dim}(R)=1$ ), then $\operatorname{FGPD}(R)=$ 1. Furthermore, if $G$ - $g l \operatorname{dim}(R)<\infty$, then $\operatorname{Gpd}_{R}(M)<\infty$ for any $R$-module $M$. Thus $G_{p p d}(M) \leqslant \operatorname{FGPD}(R)=1$. Hence $G$ - $g l \operatorname{dim}(R) \leqslant 1$. In other words, 
a one-dimensional Noetherian domain either is a G-Dedekind domain or has infinite Gorenstein global dimension.

Example 1.3. Let $\mathbb{Q}$ be the field of rational numbers and $X$ be an indeterminate. We consider the $\operatorname{ring} R=\mathbb{Q}+X^{3} \mathbb{Q}[X]$. We prove that the ideal $I=\left(X^{3}, X^{4}, X^{5}\right)$ is not G-projective. Consider the short exact sequence

$$
0 \longrightarrow I_{1} \oplus I_{2} \stackrel{\alpha}{\longrightarrow} R \oplus R \oplus R \stackrel{\beta}{\longrightarrow} I \longrightarrow 0
$$

where $I_{1}=I_{2}=I$. The homomorphisms $\alpha$ and $\beta$ are defined as follows: $\alpha\left(\left(i_{1}, i_{2}\right)\right)=\left(i_{1} X,-i_{1}, 0\right)+\left(0, i_{2} X,-i_{2}\right)$ and $\beta\left(\left(r_{1}, r_{2}, r_{3}\right)\right)=r_{1} X^{3}+r_{2} X^{4}+$ $r_{3} X^{5}$.

Next, we show that $\operatorname{Ext}_{R}^{1}(I, R) \neq 0$ and this means that $I$ is not G-projective. Let $\varphi$ be a homomorphism from $I_{1} \oplus I_{2}$ to $R$ such that $\varphi\left(\left(X^{3}, 0\right)\right)=X^{3} X^{2}=$ $X^{5}$, this is possible because $\operatorname{Hom}(I, R) \cong I^{-1}=\mathbb{Q}[X]$. If there exists a homomorphism $\theta$ from $R \oplus R \oplus R$ to $R$ such that $\varphi=\theta \alpha$, then there must exist $r, r^{\prime} \in R$ such that $X^{5}=r X^{4}-r^{\prime} X^{3}$. But this is impossible since the right hand side of this equation will never have a nonzero term of $X^{5}$.

Since the ideal $I=\left(X^{3}, X^{4}, X^{5}\right)$ is not G-projective, $R$ is not a G-Dedekind domain and $G-g l \operatorname{dim}(R)=\infty$. In fact, it can be seen from the short exact sequence $(*)$ that the Gorenstein projective dimension of $I$ can not be finite.

Recall that a Noetherian domain is said to be a 1-Gorenstein domain if its self-injective dimension is at most 1 . The following theorem is $[4$, Theorem $3.4]$.

Theorem 1.4. Let $R$ be an integral domain. Then $G$-gl $\operatorname{dim}(R) \leqslant 1$ if and only if $R$ is 1-Gorenstein.

Therefore, a domain is a G-Dedekind domain if and only if it is 1-Gorenstein.

Proposition 1.5. Let $R$ be a domain with quotient field $K$ such that $R \neq K$. Then the following statements are equivalent:

(1) $K / R$ is an injective $R$-module.

(2) $i d_{R}(R)=1$, where $i d_{R}(M)$ denotes injective dimension of $M$.

(3) For any submodule $M$ of a free module, $\operatorname{Ext}_{R}^{1}(M, R)=0$.

(4) For any ideal $I \subset R, \operatorname{Ext}_{R}^{1}(I, R)=0$.

Proof. $(1) \Rightarrow(2)$ By [20, Theorem 3.2.6], the quotient field $K$ of $R$ is an injective $R$-module. So, it can be seen from the following short exact sequence

$$
0 \longrightarrow R \longrightarrow K \longrightarrow K / R \longrightarrow 0
$$

that $i d_{R}(R) \leqslant 1$. Since $R \neq K, R$ is not a field and is not an injective module, therefore $i d_{R}(R)=1$.

$(2) \Rightarrow(1)$ It can be seen from the above short exact sequence.

$(1) \Rightarrow(3)$ Let $M$ be a submodule of some free module $F$. Consider the following exact sequence:

$$
0 \longrightarrow M \longrightarrow F \longrightarrow F / M \longrightarrow 0
$$


Because $F$ is a projective module, it can be seen that

$$
\operatorname{Ext}_{R}^{1}(M, R) \cong \operatorname{Ext}_{R}^{2}(F / M, R) .
$$

Consider also the exact sequence:

$$
0 \longrightarrow R \longrightarrow K \longrightarrow K / R \longrightarrow 0
$$

Because $K$ is an injective module, it can be seen that

$$
\operatorname{Ext}_{R}^{2}(F / M, R) \cong \operatorname{Ext}_{R}^{1}(F / M, K / R) .
$$

Therefore, $\operatorname{Ext}_{R}^{1}(M, R) \cong \operatorname{Ext}_{R}^{1}(F / M, K / R)$, and $\operatorname{Ext}_{R}^{1}(M, R)=0$ if and only if $\operatorname{Ext}_{R}^{1}(F / M, K / R)=0$. So, when $K / R$ is an injective module, $\operatorname{Ext}_{R}^{1}(M, R)=0$.

$(3) \Rightarrow(4)$ This is obvious.

$(4) \Rightarrow(1)$ Let $I$ be an ideal of $R$. Consider the following exact sequence:

$$
0 \longrightarrow I \longrightarrow R \longrightarrow R / I \longrightarrow 0
$$

Because $R$ is projective, it can be seen that $\operatorname{Ext}_{R}^{1}(I, R) \cong \operatorname{Ext}_{R}^{2}(R / I, R)$. Consider also the exact sequence:

$$
0 \longrightarrow R \longrightarrow K \longrightarrow K / R \longrightarrow 0
$$

Since $K$ is injective, it can be seen that $\operatorname{Ext}_{R}^{2}(R / I, R) \cong \operatorname{Ext}_{R}^{1}(R / I, K / R)$. So

$$
\operatorname{Ext}_{R}^{1}(I, R) \cong \operatorname{Ext}_{R}^{1}(R / I, K / R)
$$

and, $\operatorname{Ext}_{R}^{1}(I, R)=0$ if and only if $\operatorname{Ext}_{R}^{1}(R / I, K / R)=0$. Therefore, when $\operatorname{Ext}_{R}^{1}(I, R)=0$ holds for any ideal $I$ of $R, \operatorname{Ext}_{R}^{1}(R / I, K / R)=0$ also holds for any ideal $I$ of $R$. Thus $K / R$ is an injective module.

It follows from Proposition 1.5 that a Noetherian domain $R$ with quotient field $K$ is a G-Dedekind domain if and only if $K / R$ is injective.

Lemma 1.6. Let $R$ be a domain with quotient field $K$ and $I$ be an ideal of $R$. Then $\operatorname{Ext}_{R}^{1}(I, R)=0$ (for example, $I$ is G-projective) if and only if $\operatorname{Ext}_{R}^{1}(R / I, K / R)=0$.

Proof. The proof is the same as that of $(4) \Rightarrow(1)$ in Proposition 1.5.

Proposition 1.7. Let $R$ be a Noetherian domain with quotient field $K$. If every prime ideal of $R$ is G-projective, then $R$ is a G-Dedekind domain.

Proof. From the remark above, it will suffice to show that $K / R$ is injective. If we can show that $\operatorname{Ext}_{R}^{1}(M, K / R)=0$ for any finitely generated $R$-module $M$, then $K / R$ must be injective. Since $R$ is Noetherian and $M$ is a finitely generated $R$-module, by [20, Theorem 6.5 .12$]$, there exists an ascending chain of submodules of $M$ :

$$
0=M_{0} \subset M_{1} \subset M_{2} \subset \cdots \subset M_{n-1} \subset M_{n}=M
$$

such that $M_{i+1} / M_{i} \cong R / P_{i+1}$ where $P_{i}^{\prime} s$ are prime ideals of $R$ and $i=$ $0,1, \ldots, n-1$. Let $P$ be any prime ideal of $R$. Since $P$ is G-projective, by 
Lemma 1.6, $\operatorname{Ext}_{R}^{1}(R / P, K / R)=0$. Therefore $\operatorname{Ext}_{R}^{1}\left(M_{i+1} / M_{i}, K / R\right)=0$ and inductively, $\operatorname{Ext}_{R}^{1}(M, K / R)=0$.

Lemma 1.8. Let $R$ be a one-dimensional Noetherian domain with quotient field $K$ and integral closure $T$. Denote the ideal $\left(R:_{K} T\right)$ by $I$. If $J$ is an ideal of $R$ such that $J+I=R$, then $J$ is invertible.

Proof. In order to show that $J$ is invertible, it will suffice to show that $J_{P}$ is a principal ideal of $R_{P}$ for any prime ideal $P$ of $R$.

If $P$ is a prime ideal containing $I$, then $J+P=R$ and $J$ can not be inside $P$. So $J_{P}=R_{P}$ is principal.

Next we will show that $R_{P}$ is a principal ideal domain when $P$ is a prime ideal which doesn't contain $I$. Since $P$ is a prime ideal which doesn't contain $I$, there exists an element $t \in I$ but $t$ is not in $P$. Since $t T \subset R, T \subset \frac{1}{t} R \subset R_{P}$. Let $Q=P R_{P} \bigcap T$. Then $Q$ is a prime ideal of $T$ such that $Q \bigcap R=P$. Trivially, $R_{P} \subseteq T_{Q}$. Suppose $\frac{v}{s}$ is an element of $T_{Q}$ such that $v \in T$ and $s \in T-Q$. Then $t v \in R$ and $t s \in R-P$, so $\frac{v}{s}=\frac{t v}{t s} \in R_{P}$. Thus, we have proved that $R_{P}=T_{Q}$. But $T_{Q}$ is a discrete valuation domain since $T$ is a Dedekind domain. Therefore $R_{P}=T_{Q}$ is a principal ideal domain. In this case, $J_{P}$ is also a principal ideal of $R_{P}$.

Remark. If the ideal $\left(R:_{K} T\right)=0$, then the conclusion of Lemma 1.8 will become trivial. The following proposition shows that this will not happen when $T$ is a finitely generated $R$-module.

Proposition 1.9. Let $R$ be a one-dimensional Noetherian domain with quotient field $K$ and let $T$ be an overring of $R$. Then $\left(R:_{K} T\right) \neq 0$ if and only if $T$ is a finitely generated $R$-module.

Proof. If $T$ is a finitely generated $R$-module, then it can be assumed that $T$ is generated by $\frac{b_{1}}{a_{1}}, \frac{b_{2}}{a_{2}}, \ldots, \frac{b_{n}}{a_{n}}$ where $a_{i}$ 's and $b_{i}$ 's are elements of $R$. Let $s=$ $a_{1} a_{2} \cdots a_{n}$. We have $s \neq 0$ and $s \in\left(R:_{K} T\right)$. This means that $\left(R:_{K} T\right) \neq 0$.

On the other hand, if $\left(R:_{K} T\right) \neq 0$, then there is some $b \neq 0$ and $b \in$ $\left(R:_{K} T\right)$. Thus $b T$ is an ideal of $R$. Since $R$ is Noetherian, $b T$ is in fact a finitely generated $R$-module. Furthermore, by the Krull-Akizuki theorem, $T / b T$ is also a finitely generated $R$-module. Thus, it can be seen from the short exact sequence

$$
0 \longrightarrow b T \longrightarrow T \longrightarrow T / b T \longrightarrow 0
$$

that $T$ is also a finitely generated $R$-module.

Theorem 1.10. Let $R$ be a one-dimensional Noetherian domain with quotient field $K$ and integral closure $T$. Denote the ideal $\left(R:_{K} T\right)$ by $I$. Then $R$ is a $G$-Dedekind domain if and only if for any prime ideal $P$ of $R$ which contains I, $P$ is G-projective. 
Proof. If $R$ is a G-Dedekind domain, $P$ is surely G-projective. For the "if" part, by Proposition 1.7, it will suffice to prove that every prime ideal of $R$ is G-projective. If $P$ is any prime ideal which doesn't contain $I=\left(R:_{K} T\right)$, then by Lemma 1.8, $P$ is invertible and therefore G-projective. If $P$ is any prime ideal which contains $I$, then $P$ is G-projective from the hypothesis.

Corollary 1.11. Let $R$ be a one-dimensional Noetherian domain with quotient field $K$ and integral closure $T$. If $\left(R:_{K} T\right)$ is a G-projective prime ideal of $R$, then $R$ is a G-Dedekind domain.

Example 1.12. Let $R$ be the ring of $\mathbb{Q}+X^{2} \mathbb{Q}[X]$ where $\mathbb{Q}$ is the field of rational numbers and $X$ an indeterminate. We prove that the ideal $I=\left(X^{2}, X^{3}\right)$ is $\mathrm{G}$-projective. Consider the short exact sequence

$$
0 \longrightarrow I \stackrel{\alpha}{\longrightarrow} R \oplus R \stackrel{\beta}{\longrightarrow} I \longrightarrow 0
$$

where $\alpha(i)=(i X,-i)$ and $\beta\left(\left(r_{1}, r_{2}\right)\right)=r_{1} X^{2}+r_{2} X^{3}$.

In the following, we will prove that $\operatorname{Ext}_{R}^{1}(I, R)=0$ and this will show from [5, Proposition 2.9] that $I$ is G-projective (in fact it is strongly G-projective).

Applying the functor $\operatorname{Hom}(-, R)$ to the short exact sequence $(* *)$, the following exact sequence is obtained:

$$
0 \longrightarrow \operatorname{Hom}(I, R) \stackrel{\beta^{*}}{\longrightarrow} \operatorname{Hom}(R \oplus R, R) \stackrel{\alpha^{*}}{\longrightarrow} \operatorname{Hom}(I, R) \longrightarrow \operatorname{Ext}_{R}^{1}(I, R) \longrightarrow 0
$$

Note that $\operatorname{Ext}_{R}^{1}(I, R)=0$ is equivalent to the fact that $\alpha^{*}$ is a surjective map. In other words, if for any homomorphism $\psi$ from $I$ to $R$, there exists a homomorphism $\theta$ from $R \oplus R$ to $R$ such that $\psi=\theta \alpha$, then $\operatorname{Ext}_{R}^{1}(I, R)=$ 0 . Since $\operatorname{Hom}(I, R) \cong I^{-1}=\mathbb{Q}[X]$, we can assume that $\psi(i)=i f(X)=$ $i q_{1} X+i g(X)$ for some $f(X)=q_{1} X+g(X) \in \mathbb{Q}[X]$ and $g(X) \in R$. Now, let $\theta\left(r_{1}, r_{2}\right)=q_{1} r_{1}-r_{2} g(X)$. Then

$$
\theta \alpha(i)=\theta((i X,-i))=q_{1} i X+i g(X)=i q_{1} X+i g(X)=i f(X)=\psi(i) .
$$

Therefore $\operatorname{Ext}_{R}^{1}(I, R)=0$.

Note that the integral closure of $R$ in its field of quotients is $\mathbb{Q}[X]$ and $I=(R: \mathbb{Q}[X])$. We also notice that $I$ is a prime ideal of $R$. Therefore, by Corollary 1.11, $R$ is in fact a G-Dedekind domain. Since $R$ is not integrally closed, $R$ is not a Dedekind domain.

\section{Overrings of G-Dedekind domains}

It is well known that any overring of a Dedekind domain is still a Dedekind domain. It is natural to ask whether overrings of a G-Dedekind domain are G-Dedekind domains or not? The following example shows that this is not necessarily the case.

Example 2.1. Let $D=\mathbb{Q}[y, z]$, where $y, z$ are two indeterminates and $\mathbb{Q}$ is the field of rational numbers. Then, the ring

$R=\left\{f \in \mathbb{Q}[x] \mid f=q_{0}+q_{3} X^{3}+q_{4} X^{4}+q_{6} X^{6}+\cdots+q_{k} X^{k}, q_{i} \in \mathbb{Q}\right\} \cong D /\left(y^{4}-z^{3}\right)$ 
is a G-Dedekind domain. The elements in $R$ are those polynomials whose terms of $X, X^{2}$ and $X^{5}$ are zero. To see that $R$ is a G-Dedekind domain, we just need to notice that $g l \cdot \operatorname{dim}(D)=2$ and $R$ is not a QF-ring. Then, an application of $\left[13\right.$, Corollary 2.6] gives the result. But the ring $\mathbb{Q}+X^{3} \mathbb{Q}[X]$ is an overring of $R$ which is not a G-Dedekind domain. From this fact, we later show that $R$ is not a Warfield domain.

In order to give some definitions, we need to consider the following evaluation map:

$$
\Phi_{M}: M \longrightarrow M^{* *}=\operatorname{Hom}_{R}\left(\operatorname{Hom}_{R}(M, R), R\right)
$$

where $M$ is an $R$-module and $R$ is a domain. The following definitions can be found in [19].

If the evaluation map $\Phi_{M}$ is injective, then $M$ is said to be a torsionless module. If the evaluation map $\Phi_{M}$ is bijective, then $M$ is said to be reflexive; $R$ is called reflexive if all torsionless $R$-modules $M$ of finite rank are reflexive; $R$ is called divisorial if all the ideals (i.e., torsionless modules of rank 1) of $R$ are reflexive; $R$ is said to be totally divisorial if all the overrings of $R$ are divisorial; $R$ is said to be totally reflexive if all the overrings of $R$ are reflexive.

The following theorem is [19, Theorem 3.1]. From this, it can be seen that a Noetherian domain is divisorial if and only if it is reflexive.

Theorem 2.2. For a Noetherian domain $R$ with quotient field $K$, the following conditions are equivalent:

(1) $R$ is divisorial;

(2) $K / R$ is an injective $R$-module;

(3) $R$ has Krull dimension 1 and $(R: P)$ can be generated by 2 elements, for every maximal ideal $P$ of $R$;

(4) $R$ is reflexive.

Since the statement (2) is equivalent to saying that $R$ is a G-Dedekind domain for a Noetherian domain $R$, we also have that a Noetherian domain is divisorial if and only if it is a G-Dedekind domain. The following two theorems are [19, Theorem 6.3] and [19, Theorem 6.4], respectively.

Theorem 2.3. A domain $R$ is a Warfield domain if and only if it is totally reflexive.

Theorem 2.4. For a Noetherian domain $R$ the following conditions are equivalent:

(1) $R$ is a Warfield domain.

(2) all the ideals of $R$ are 2-generated.

If a one-dimensional Noetherian domain $R$ is a Warfield domain, then any overring of $R$ must be divisorial since $R$ is totally reflexive. Furthermore, by the Krull-Akizuki theorem, any overring of $R$ is also Noetherian. Therefore, any overring of $R$ must be a G-Dedekind domain. Thus the ring we give at the beginning of this section is not a Warfield domain. On the other hand, if 
any overring of a G-Dedekind domain $R$ is still a G-Dedekind domain, then $R$ must be totally divisorial and further, totally reflexive since it is a Noetherian domain. Therefore, by the above two theorems or [3, Theorem 7.3], $R$ must be a Warfield domain and all the ideals of $R$ are 2-generated (the definition of a Warfield domain can be seen in [19]). Next, we will prove that the G-Dedekind domain $\mathbb{Q}+X^{2} \mathbb{Q}[X]$ is a Warfield domain. From this example and the example before, the following relations can be given:

Dedekind domain $\subsetneq$ Noetherian Warfield domain $\subsetneq$ G-Dedekind domain.

\subsection{Ideals of the ring $\mathbb{Q}+X^{2} \mathbb{Q}[X]$}

Proposition 2.5. Let $f$ and $g$ be two elements of the ring $R=\mathbb{Q}+X^{2} \mathbb{Q}[X]$. If $f \mathbb{Q}[X]+g \mathbb{Q}[X]=\mathbb{Q}[X]$, then $f R+g R=R$.

Proof. Suppose $f u+g v=1$ where $u, v \in \mathbb{Q}[X]$. Then, we can assume that $u=u^{\prime}+t_{1} X$ and $v=v^{\prime}+t_{2} X$ where $u^{\prime}, v^{\prime} \in R$ and $t_{1}, t_{2} \in \mathbb{Q}$. So,

$$
f u+g v=1 \Rightarrow f u^{\prime}+g v^{\prime}=1-X\left(t_{1} f+t_{2} g\right) .
$$

Since $f u^{\prime}+g v^{\prime} \in R, 1-X\left(t_{1} f+t_{2} g\right) \in R$. So $1+X\left(t_{1} f+t_{2} g\right) \in R$. Let $h=1+X\left(t_{1} f+t_{2} g\right)$. Then $f u^{\prime} h+g v^{\prime} h=1-X^{2}\left(t_{1} f+t_{2} g\right)^{2}$. A simple observation will give that $f R+g R=R$.

Proposition 2.6. Let $J$ be an ideal of the ring $R=\mathbb{Q}+X^{2} \mathbb{Q}[X]$ such that $J+I=R$ where $I=\left(X^{2}, X^{3}\right)$. Then $J$ is invertible and can be factored uniquely into a product of prime ideals.

Proof. Notice that $I=(R: \mathbb{Q}[X])$ and $\mathbb{Q}[X]$ is the integral closure of $R$. An application of Lemma 1.8 will give that $J$ is invertible. For the second claim, we will prove that any ideal $J$ with the property that $J+I=R$ can be factored uniquely into a product of prime ideals.

Suppose the set $\mathbb{M}$ of those ideals which do not have factorization is not empty. As $R$ is Noetherian, $\mathbb{M}$ admits a maximal element $L$. This can not be a prime ideal and hence must be contained in a maximal ideal $P$. Since $L+I=R$, we have $P+I=R$ and $P$ is invertible by Lemma 1.8. Thus $P$ is divisorial. So we have $P^{-1} \supsetneqq R$ and $L \neq P$. We have the following: $L \subseteq L P^{-1} \subseteq P P^{-1}=R$. From the invertibility of $L$ and $P$ we get that $L P^{-1} \neq L$ and $L P^{-1} \neq P P^{-1}$. Since $L P^{-1}$ properly contains $L, L P^{-1}$ can be uniquely factored and so $L$ can be factored too. This contradiction shows that $\mathbb{M}$ is empty. Therefore every ideal which is prime to $I$ can be factored into a product of prime ideals. Notice that prime factors are also relatively prime to $I$, and so we get the invertibility of these factors and the uniqueness of the factorization.

Remark. Let $R$ be a one-dimensional Noetherian domain with $T$ its integral closure in the quotient field $K$. It can be seen from the above proof that, if $J$ is an ideal of $R$ such that $J+\left(R:_{K} T\right)=R$, then $J$ is invertible and can be factored uniquely into a product of some prime ideals. 
Example 2.7. This example is to show that invertible ideals of $R=\mathbb{Q}+$ $X^{2} \mathbb{Q}[X]$ are not necessarily principal. Let $J=\left(1+X^{2}+X^{4}, 1+X^{3}\right)$. Then $J+I=R$ since $I=\left(X^{2}, X^{3}\right)$ is a maximal ideal of $R$ and $J$ is not contained in $I$. So, by Proposition 2.6, $J$ is invertible. Since

$$
\begin{aligned}
J \mathbb{Q}[X] & =\left(1+X^{2}+X^{4}\right) \mathbb{Q}[X]+\left(1+X^{3}\right) \mathbb{Q}[X] \\
& =\left(1-X+X^{2}\right)\left(\left(1+X+X^{2}\right) \mathbb{Q}[X]+(1+X) \mathbb{Q}[X]\right) \\
& =\left(1-X+X^{2}\right) \mathbb{Q}[X],
\end{aligned}
$$

we have that $J \neq R$. Next, we show that $J$ is not a principal ideal.

First, we prove that $1+X^{2}+X^{4}$ is an irreducible elements of $R$. To see this fact, we just need to notice that $1+X^{2}+X^{4}=\left(1-X+X^{2}\right)\left(1+X+X^{2}\right)$ and the ring $\mathbb{Q}[X]$ is a UFD (uniquely factorization domain).

Second, we show that $1+X^{3}$ is not in the ideal $\left(1+X^{2}+X^{4}\right)$. Otherwise, $1+X^{3}=\left(1+X^{2}+X^{4}\right) g(X) \Rightarrow 1+X=\left(1+X+X^{2}\right) g(X)$. But this is impossible since $1+X$ and $1+X+X^{2}$ are irreducible elements of the UFD $\mathbb{Q}[X]$.

If $J$ is principal, then $J=\left(1+X^{2}+X^{4}\right)$ since $1+X^{2}+X^{4}$ is irreducible. But the second step shows that this can not happen.

Next, we will prove that every ideal of the $\operatorname{ring} R=\mathbb{Q}+X^{2} \mathbb{Q}[X]$ can be generated by two elements. This means that $R$ is a Warfield domain.

Lemma 2.8. Let $R^{\prime}=\mathbb{Q}\left[X^{2}\right]$. Then $R^{\prime}$ is a principal ideal domain and $R=$ $\mathbb{Q}+X^{2} \mathbb{Q}[X]$ is a free $R^{\prime}$-module of rank 2 .

Proof. Since $R^{\prime}=\mathbb{Q}\left[X^{2}\right] \cong \mathbb{Q}[Y]$ where $Y$ is an indeterminate and $\mathbb{Q}[Y]$ is a principal ideal domain, $R^{\prime}$ is also a principal ideal domain. For the second claim, we can find a basis of the $R^{\prime}$-module $R$ : 1 and $X^{3}$. Let $f$ be any element of $R$. Then, the odd degree of $X$ in terms of $f$ must be larger than or equal to 3. So, $f=r_{1}^{\prime}+r_{2}^{\prime} X^{3}$ for some $r_{1}^{\prime}, r_{2}^{\prime} \in R^{\prime}$. The uniqueness of this expression can be explained as follows: $f=0$ if and only if both the sum of even degree terms and the sum of odd degree terms of $f$ are zero.

Theorem 2.9. Every ideal of the ring $R=\mathbb{Q}+X^{2} \mathbb{Q}[X]$ can be generated by two elements.

Proof. Let $R^{\prime}=\mathbb{Q}\left[X^{2}\right]$. Then, every ideal of the ring $R=\mathbb{Q}+X^{2} \mathbb{Q}[X]$ is in fact an $R^{\prime}$-module. By Lemma $2.8, R=\mathbb{Q}+X^{2} \mathbb{Q}[X]$ is a free $R^{\prime}$-module of rank 2. Therefore, every ideal of $R$ is in fact a submodule a free $R^{\prime}$-module of rank 2. Again by Lemma $2.8, R^{\prime}=\mathbb{Q}\left[X^{2}\right]$ is a principal ideal domain. So any ideal $J$ of $R$ is also a free $R^{\prime}$-module and the rank of $J$ is at most 2 . Since, as an $R^{\prime}$-module, any ideal $J$ of $R$ can be generated by two elements, $J$ can also be generated by two elements as an $R$-module. 


\subsection{Some properties of Noetherian Warfield domains}

In what follows, we will give a direct proof to show that the Krull dimension of any Noetherian Warfield domain is at most 1 and prove that any ideal of a Noetherian Warfield domain is 2-strongly Gorenstein projective.

Proposition 2.10. Let $R$ be a Noetherian local ring and $M$ be its maximal ideal. If both $M$ and $M^{2}$ can be generated by two elements, then the Krull dimension of $R$ is at most 1.

Proof. We prove that $M$ is the minimal prime ideal over some principal ideal of $R$. Suppose that $M=\left(x_{1}, x_{2}\right)$, then $M^{2}=\left(x_{1}^{2}, x_{1} x_{2}, x_{2}^{2}\right)$. Since $M^{2}$ can be generated by two elements, the dimension of the vector space $M^{2} / M^{3}$ over the field $R / M$ is at most two. Therefore, there exist $\bar{a}, \bar{b}, \bar{c} \in R / M$, not all of them be zero, such that $\bar{a}{\overline{x_{1}}}^{2}+\bar{b} \overline{x_{1}} \overline{x_{2}}+\bar{c}{\overline{x_{2}}}^{2}=0$ where $\overline{x_{i}} \overline{x_{j}} \in M^{2} / M^{3}$. This means that $a x_{1}^{2}+b x_{1} x_{2}+c x_{2}^{2} \in M^{3}$. Next we will prove that there exist two elements $\overline{a_{1}}, \overline{a_{2}} \in R / M$ such that $\bar{a}{\overline{a_{1}}}^{2}+\bar{b} \overline{a_{1}} \overline{a_{2}}+\bar{c}{\overline{a_{2}}}^{2} \neq 0$.

Case 1: $|R / M|=2$. In this case, the symbol $(u, v, w)$ means an ordered triple where $u, v, w \in R / M$. Considering the symmetry, we only need to discuss the following five situations:

$$
(\bar{a}, \bar{b}, \bar{c})=(1,0,0) ;(0,1,0) ;(1,1,0) ;(1,0,1) ;(1,1,1) .
$$

If $(\bar{a}, \bar{b}, \bar{c})=(1,0,0)$ or $(1,1,0)$ or $(1,0,1)$, then we let $\overline{a_{1}}=1, \overline{a_{2}}=0$ and get that $\bar{a}{\overline{a_{1}}}^{2}+\bar{b} \overline{a_{1}} \overline{a_{2}}+\bar{c}{\overline{a_{2}}}^{2}=1 \neq 0$. If $(\bar{a}, \bar{b}, \bar{c})=(0,1,0)$ or $(1,1,1)$, then we let $\overline{a_{1}}=1, \overline{a_{2}}=1$ and get that $\bar{a}{\overline{a_{1}}}^{2}+\bar{b} \overline{a_{1}} \overline{a_{2}}+\bar{c}{\overline{a_{2}}}^{2}=1 \neq 0$.

Case 2: $|R / M|>2$. Let $\overline{a_{2}}=1$. Then $\bar{a}{\overline{a_{1}}}^{2}+\bar{b} \overline{a_{1}} \overline{a_{2}}+\bar{c} \overline{a_{2}}{ }^{2}=\bar{a} \overline{a_{1}}{ }^{2}+\bar{b} \overline{a_{1}}+\bar{c}$. Note that the quadratic equation $\bar{a} y^{2}+\bar{b} y+\bar{c}=0$ has at most two roots in $R / M$. So if we let $\overline{a_{1}}$ be an element other than these roots, we have that $\bar{a}{\overline{a_{1}}}^{2}+\bar{b} \overline{a_{1}} \overline{a_{2}}+\bar{c}{\overline{a_{2}}}^{2} \neq 0$.

Without loss of generality, we can assume that $\overline{a_{2}}=\overline{1}$. So, we have $\bar{a}{\overline{a_{1}}}^{2}+$ $\bar{b} \overline{a_{1}}+\bar{c} \neq 0$. This means that $a a_{1}^{2}+b a_{1}+c$ is a unit of $R$. Let $x=x_{1}-a_{1} x_{2}$. We have

$a x_{1}^{2}+b x_{1} x_{2}+c x_{2}^{2}=a\left(x+a_{1} x_{2}\right)^{2}+b\left(x+a_{1} x_{2}\right) x_{2}+c x_{2}^{2}=\left(a a_{1}^{2}+b a_{1}+c\right) x_{2}^{2}+r x$ for some $r \in R$. This means that $x_{2}^{2} \in R x+M^{3}$. Since

$$
M^{2}=\left(x_{1}^{2}, x_{1} x_{2}, x_{2}^{2}\right)=\left(\left(x+a_{1} x_{2}\right)^{2},\left(x+a_{1} x_{2}\right) x_{2}, x_{2}^{2}\right) \subset R x+R x_{2}^{2},
$$

we have $M^{2} \subset R x+M^{3}$, and hence $M^{2} \subset R x+M^{k}$ for all integers $k \geqslant 3$. Therefore $M^{2} \subset \bigcap_{i=3}^{\infty}\left(R x+M^{i}\right)=R x$ by [15, p. 55, Exercise $\left.7(\mathrm{~d})\right]$. Since some power of $M$ is inside $R x, M$ must be the minimal prime ideal over the principal ideal $R x$. Therefore, by [20, Theorem 6.6.2], the Krull dimension of $R$ is at most 1 .

Definition 2.11 ([6, Definition 2.1]). A module $M$ is said to be $n$-strongly Gorenstein projective ( $n$-SG-projective), if there exists an exact sequence of modules $0 \longrightarrow M \longrightarrow P_{n} \longrightarrow \cdots \longrightarrow P_{1} \longrightarrow M \longrightarrow 0$, where each $P_{i}$ is 
projective such that $\operatorname{Hom}(-, Q)$ leaves the sequence exact whenever $Q$ is a projective module.

Lemma 2.12. Let $R$ be a domain. If $I=(a, b)$ and $J=(c, d)$ are two fractional ideals of $R$ such that $\frac{b}{a}=\frac{d}{c}$, then $I \cong J$.

Proof. Denote $\frac{b}{a}=\frac{d}{c}$ by $\alpha$. Then, $I=a(1, \alpha) \cong(1, \alpha) \cong c(1, \alpha)=J$.

Lemma 2.13. Let $R$ be a domain. If $I=(a, b)$ is an ideal of $R$ which is not principal, then there exists a short exact sequence

$$
0 \longrightarrow I^{-1} \longrightarrow R \oplus R \longrightarrow I \longrightarrow 0 \text {. }
$$

Proof. Since $I$ is not principal, neither $\frac{a}{b}$ nor $\frac{b}{a}$ is inside $R$. So, by Lemma 2.12 , we can assume that $I \cong(1, \beta)$ where $(1, \beta) \supset R$ is a fractional ideal of $R$. Since $(1, \beta) \supset R$, we have $(1, \beta)^{-1} \subset R$ is an ideal of $R$. Denote $(1, \beta)$ by $J$. Then $J^{-1}=\{x \in K \mid x(1, \beta) \in R\}=\{x \in R \mid x \beta \in R\}$. Now, we can construct the following sequence

$$
0 \longrightarrow J^{-1} \stackrel{f}{\longrightarrow} R \oplus R \stackrel{g}{\longrightarrow} J \longrightarrow 0
$$

where $f(x)=(x \beta,-x), x \in J^{-1}$ and $g\left(\left(r_{1}, r_{2}\right)\right)=r_{1}+r_{2} \beta,\left(r_{1}, r_{2}\right) \in R \oplus R$. The injectivity of $f$ and the surjectivity of $g$ are obvious. That $\operatorname{Im} f \subset \operatorname{ker} g$ can be seen from the construction. In order to prove the exactness of this sequence, we only need to confirm that $\operatorname{ker} g \subset \operatorname{Im} f$. Suppose $g\left(\left(r_{1}, r_{2}\right)\right)=r_{1}+r_{2} \beta=0$. Then $r_{2} \beta=-r_{1} \in R$ and $r_{2} \in R$ and this means that $r_{2} \in(1, \beta)^{-1}=J^{-1}$. Thus $\left(r_{1}, r_{2}\right)=\left(-r_{2} \beta, r_{2}\right)=f\left(-r_{2}\right) \in \operatorname{Im} f$.

Finally, since $I \cong J$, it can be seen that $I^{-1} \cong \operatorname{Hom}(I, R) \cong \operatorname{Hom}(J, R) \cong$ $J^{-1}$. Thus, we have a short exact sequence

$$
0 \longrightarrow I^{-1} \longrightarrow R \oplus R \longrightarrow I \longrightarrow 0 .
$$

Corollary 2.14. Let $R$ be a $G$-Dedekind domain and let $I$ be an ideal of $R$ which is generated by two elements. If $I^{-1} \cong I$, then $I$ is strongly G-projective.

Proof. If $I$ is principal, it is surely strongly G-projective. In the case $I$ is not principal, just apply Lemma 2.13.

Example 2.15. Consider the ideal $I=\left(X^{2}, X^{3}\right)$ of the $\operatorname{ring} R=\mathbb{Q}+X^{2} \mathbb{Q}[X]$. Since $I^{-1}=\mathbb{Q}[X]=(1, X), I^{-1} \cong I$. By Corollary 2.14, $I$ is strongly Gprojective. Similarly, ideals of the form $\left(X^{n}, X^{n+1}\right)(n \geqslant 2)$ are also strongly G-projective.

Lemma 2.16. Let $R$ be a domain. If $I$ is a divisorial ideal of $R$ such that both $I$ and $I^{-1}$ can be generated by two elements, then $I$ is G-projective.

Proof. If $I$ is principal, it is certainly G-projective. So, it can be assumed that $I$ is not principal. By Lemma 2.13, there exists the following exact sequence:

$$
0 \longrightarrow I^{-1} \stackrel{f}{\longrightarrow} R \oplus R \stackrel{g}{\longrightarrow} I \longrightarrow 0 .
$$


Without loss of generality, we can assume that $I=(1, a)$ and $f(x)=(x a,-x)$, $x \in I^{-1}$ and $g\left(\left(r_{1}, r_{2}\right)\right)=r_{1}+r_{2} a,\left(r_{1}, r_{2}\right) \in R \oplus R$. Next, we prove that $\operatorname{Ext}_{R}^{1}(I, R)=0$. Let $\alpha$ be any homomorphism from $I^{-1}$ to $R$. If we can find a homomorphism $\beta$ from $R \oplus R$ to $R$ such that $\alpha=\beta f$, then the homomorphism $\operatorname{Hom}(R \oplus R, R) \longrightarrow \operatorname{Hom}\left(I^{-1}, R\right)$ is surjective and it can be seen from the long exact sequence

$$
0 \rightarrow \operatorname{Hom}(I, R) \rightarrow \operatorname{Hom}(R \oplus R, R) \rightarrow \operatorname{Hom}\left(I^{-1}, R\right) \rightarrow \operatorname{Ext}_{R}^{1}(I, R) \rightarrow 0
$$

that $\operatorname{Ext}_{R}^{1}(I, R)=0$. Since $I=I_{v} \cong \operatorname{Hom}\left(I^{-1}, R\right)$, we can assume that $\alpha(x)=x\left(r_{1}^{\prime}+r_{2}^{\prime} a\right)$ for some $r_{1}^{\prime}, r_{2}^{\prime} \in R$. Let $\beta\left(\left(r_{1}, r_{2}\right)\right)=r_{1} r_{2}^{\prime}-r_{2} r_{1}^{\prime}$. Then $\alpha(x)=x\left(r_{1}^{\prime}+r_{2}^{\prime} a\right)=r_{1}^{\prime} x+r_{2}^{\prime} a x=\beta((x a,-x))=\beta f(x)$. Similarly, we also have a short exact sequence

$$
0 \longrightarrow I \stackrel{f}{\longrightarrow} R \oplus R \stackrel{g}{\longrightarrow} I^{-1} \longrightarrow 0
$$

and $\operatorname{Ext}_{R}^{1}\left(I^{-1}, R\right)=0$. Combining these two short exact sequences, we get an exact sequence:

$$
0 \longrightarrow I \longrightarrow R \oplus R \longrightarrow R \oplus R \stackrel{g}{\longrightarrow} I \longrightarrow 0 .
$$

Note that the kernel of $g$ is isomorphic to $I^{-1}$. Since $\operatorname{Ext}_{R}^{1}\left(I^{-1}, R\right)=0$ and $\operatorname{Ext}_{R}^{1}(I, R)=0, I$ must be G-projective (in fact, it is 2-SG-projective).

Corollary 2.17. Let $R$ be a domain in which every ideal can be generated by two elements. Then every divisorial ideal is G-projective.

Remark. By Proposition 2.10, if $R$ is a ring in which every ideal can be generated by two elements, the Krull dimension of $R$ is at most 1 . In fact, it had been proved in [8] that, if there exists a fixed upper bound for the number of generating set of any ideal of $R$, then $R$ is Noetherian and the Krull dimension of $R$ is at most 1 . Therefore, if $R$ is a domain in which every ideal can be generated by two elements, then, by [20, Theorem 8.1.5], every nonzero prime ideal of $R$ is divisorial since it is minimal over some principal ideal. Thus, by Corollary 2.17, every prime ideal of $R$ is G-projective. Furthermore, by Proposition 1.7, $R$ is a G-Dedekind domain.

Lemma 2.18. Let $R$ be a G-Dedekind domain and let $I$ be a nonzero ideal of $R$. Then $I$ is principal if and only if $I^{-1}$ is principal.

Proof. If $I^{-1}$ is principal, then $I^{-1} \cong R$. So $I=\left(I^{-1}\right)^{-1} \cong \operatorname{Hom}\left(I^{-1}, R\right) \cong$ $\operatorname{Hom}(R, R) \cong R$. This means that $I$ must be principal. On the other hand, if $I=(x)$ is principal, then $I^{-1}=\left(x^{-1}\right)$ is principal.

Lemma 2.19. Let $R$ be a Noetherian Warfield domain and let $I=(a, b)$ be a fractional ideal of $R$ which is not principal. Then, there exists a short exact sequence

$$
0 \longrightarrow I \longrightarrow R \oplus R \longrightarrow I^{-1} \longrightarrow 0
$$


Proof. Without loss of generality, we can assume that $I \subset R$ is an ideal of $R$. Since $I$ is not principal, by Lemma 2.18, neither is $I^{-1}$ principal. We can further assume that $I^{-1} \cong(1, \beta)$ where $(1, \beta) \supset R$ is a fractional ideal of $R$. By noticing that $I$ is divisorial, the rest of the proof is just as that of Theorem 2.13 .

Theorem 2.20. Let $R$ be a Noetherian Warfield domain. Then every ideal of $R$ is 2-SG-projective.

Proof. Let $I$ be an ideal of $R$. If $I$ is principal, it is certainly projective and therefore 2-strongly G-projective. So, we can assume that $I$ is not principal. In this case, neither $I$ nor $I^{-1}$ is principal by Lemma 2.18. Further, by Lemma 2.19 , we have two exact sequences

$$
0 \longrightarrow I \longrightarrow R \oplus R \longrightarrow I^{-1} \longrightarrow 0
$$

and

$$
0 \longrightarrow I^{-1} \longrightarrow R \oplus R \longrightarrow I \longrightarrow 0 .
$$

Combining these two exact sequences, we get an exact sequence:

$$
(* * * *) \quad 0 \longrightarrow I \longrightarrow R \oplus R \longrightarrow R \oplus R \longrightarrow I \longrightarrow 0 \text {. }
$$

Since $R$ is already a G-Dedekind domain, every ideal of $R$ is G-projective and we have $\operatorname{Ext}_{R}^{1}(I, Q)=0$ and $\operatorname{Ext}_{R}^{1}\left(I^{-1}, Q\right)=0$. Thus, $\operatorname{Hom}(-, Q)$ leaves the sequence $(* * * *)$ exact whenever $Q$ is a projective module. Therefore, $I$ is 2-SG-projective.

\subsection{2-DVRs and Noetherian Warfield domains}

In this subsection, we will give a characterization of Noetherian Warfield domains. For this purpose, we give a definition for a special kind of Noetherian local domain.

Definition 2.21. Let $R$ be a Noetherian local domain. Then $R$ is called a 2 -DVR if the maximal ideal of $R$ is strongly G-projective.

Remark. Comparing with the definition of a DVR (discrete valuation ring), a 2-DVR is a generalization of a DVR. A domain $R$ is a Dedekind domain if and only if $R_{M}$ is a DVR for any maximal ideal $M$. In the following, we will prove that a Noetherian domain $R$ is a Warfield domain if and only if $R_{M}$ is a 2-DVR for any maximal ideal $M$ of $R$. In order to give a characterization of a 2-DVR, we need the following three lemmas. Some tricks are adopted from [17].

Lemma 2.22. Let $R$ be a domain with quotient field $K$ and $I$ be a nonzero ideal of $R$. If the ring $R_{1}=\left(I:_{K} I\right)$ is divisorial, then $I$ is a projective $R_{1}$-module.

Proof. For any ideal $J$ of $R_{1}$, we define $J^{-1_{1}}=\left\{x \in K \mid x J \subset R_{1}\right\}$. If we can prove that $I I^{-1_{1}}=R_{1}$, then $I$ is a projective $R_{1}$-module. Suppose $I I^{-1_{1}} \neq R_{1}$. Thus there exists a maximal ideal $P$ of $R_{1}$ such that $I I^{-1_{1}} \subset P$. Hence

$$
P^{-1_{1}} I I^{-1_{1}} \subset P^{-1_{1}} P \subset R_{1},
$$


and therefore $P^{-1_{1}} I \subset\left(I^{-1_{1}}\right)^{-1_{1}}$. Since $R_{1}$ is divisorial, we have $\left(I^{-1_{1}}\right)^{-1_{1}}=$ I. Thus $P^{-1_{1}} \subset R_{1}$, and it follows that $P^{-1_{1}}=R_{1}$. But $R_{1}$ is divisorial, and therefore $\left(P^{-1_{1}}\right)^{-1_{1}}=P$. This contradiction shows that $I$ is a projective $R_{1}$-module.

Lemma 2.23. Let $R$ be a Noetherian domain with quotient field $K$ and $I$ be a nonzero ideal of $R$. Then the ring $R_{1}=\left(I:_{K} I\right)$ is a finitely generated R-module.

Proof. Take a nonzero element $a \in I$. Then $a R_{1}$ is a nonzero ideal of $R$ which is contained in $I$. Since $R$ is Noetherian, $a R_{1}$ is a finitely generated $R$-module. Thus $R_{1}$ is also a finitely generated $R$-module.

Lemma 2.24. Let $R$ be a local Noetherian domain with quotient field $K$ and $I$ be an ideal of $R$. If the ring $R_{1}=\left(I:_{K} I\right)$ is divisorial, then $R_{1}$ is isomorphic to $I$ as $R$-modules.

Proof. Since, by Lemma $2.23, R_{1}$ is a finitely generated module over the local domain $R, R_{1}$ is a semi-local domain, and finitely generated projective $R_{1}$ modules are free by [9, Chapter VI, Theorem 1.11]. Because, by Lemma 2.22, $I$ is a projective $R_{1}$-module, $I$ must be a principal ideal of $R_{1}$. This means that $I=R_{1} a$ for some $a \in I$. Therefore $I \cong R_{1}$ as $R$-modules.

Theorem 2.25. Let $R$ be a local Noetherian domain with quotient field $K$ and $M$ be the maximal ideal of $R$. The following statements are equivalent:

(1) $R$ is a 2-DVR.

(2) Every ideal of $R$ can be generated by two elements.

(3) $M$ can be generated by two elements and both $R$ and $R_{1}=\left(M:_{K} M\right)$ are divisorial domains.

Proof. (1) $\Rightarrow(2)$ If $M$ is projective, then $M$ must be principal since $R$ is local. This means that every ideal of $R$ is also principal. So we can assume that $M$ is not projective. Denote the ring $\left(M:_{K} M\right)$ by $R_{1}$. Then $M^{-1}=R_{1}$.

First, we prove that, as an $R_{1}$-ideal, $M$ is principal. Since $M$ is a strongly G-projective $R$-module, there exists a short exact sequence

$$
0 \longrightarrow M \longrightarrow P \longrightarrow M \longrightarrow 0 \text {. }
$$

Since $R$ is a local ring, the middle projective module $P$ of rank 2 is a free module by [1, Corollary 26.7]. Thus we surely have a short exact sequence

$$
0 \longrightarrow M \longrightarrow R \oplus R \longrightarrow M \longrightarrow 0 \text {. }
$$

It can be seen from this short exact sequence that $M$ is generated by two elements. Thus, by Lemma 2.13, there also exists the following short exact sequence

$$
0 \longrightarrow M^{-1} \longrightarrow R \oplus R \longrightarrow M \longrightarrow 0
$$


Therefore, there exists the following commutative diagram with exact rows.

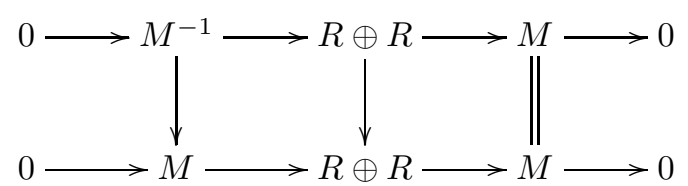

So considering the mapping cone complex or by Schanuel's Lemma, we have $M^{-1} \oplus R \oplus R \cong M \oplus R \oplus R$. Since $R$ is a local ring, by [20, Theorem 2.10.10], $M^{-1} \cong M$. Let $\psi: M^{-1} \rightarrow M$ be the isomorphism. We will prove that $M=M^{-1} \psi(1)\left(1 \in M^{-1}\right.$ follows from the fact that $M^{-1}=\left(M:_{K} M\right)$ is a ring). It is obvious that $M^{-1} \psi(1) \subset M^{-1} M=M$. Let $x \in M$ be any element of $M$. Then $x=\psi\left(\frac{r_{2}}{r_{1}}\right)$ for some $\frac{r_{2}}{r_{1}} \in M^{-1}$. It can be seen that $x=\frac{r_{2}}{r_{1}} \psi(1)$. So $M \subset M^{-1} \psi(1)$. This means that $M=M^{-1} \psi(1)=R_{1} \psi(1)$ is a principal ideal of $R_{1}$.

We have just shown that there exists an element $a \in M$ such that $M=R_{1} a$. Therefore we have the relations

$$
M^{2}=M a \subsetneq R a \subsetneq M \subsetneq R .
$$

Thus $a$ is a system of parameters, and so $\operatorname{dim}(R)=1$ by [18, Theorem 14.1].

Note that $R$ is a one-dimensional Noetherian domain. Thus if $I$ is a nonzero ideal of $R, R / I$ has finite length, which we shall denote by $l(R / I)$. Since $M$ is generated by two elements, $l\left(M / M^{2}\right)=2$. Thus $l\left(R / M^{2}\right)=3$. It can be seen from above relations that $l(R / R a)=2$.

Next we prove that any non-principal ideal $I$ of $R$ is also an ideal of $R_{1}$. It can be seen by Proposition 1.7 that $R$ is a G-Dedekind domain and therefore every ideal of $R$ is divisorial. Since $I$ is not invertible, $I I^{-1} \subset M$. Therefore

$$
R_{1} I I^{-1} \subset R_{1} M=M .
$$

Hence $R_{1} I \subset\left(I^{-1}\right)^{-1}=I$.

Let $I$ be a non-principal ideal of $R$. Then $I$ is also an ideal of $R_{1}$ and $M I=R_{1} a I=a I$. Hence $R a / M I=R a / I a \cong R / I$. Considering the following two short exact sequences

$$
0 \longrightarrow R a / M I \longrightarrow R / M I \longrightarrow R / R a \longrightarrow 0
$$

and

$$
0 \longrightarrow I / M I \longrightarrow R / M I \longrightarrow R / I \longrightarrow 0,
$$

it can be seen that $l(I / M I)=l(R / R a)=2$. This means that $I$ can be generated by two elements.

$(2) \Rightarrow(3)$ By $[19$, Theorem 6.4], $R$ is a Noetherian Warfield domain.

$(3) \Rightarrow(1)$ If $M$ is invertible, then $M$ is projective and therefore surely strongly G-projective. So we can assume that $M$ is not invertible. Since $M \subset$ $M M^{-1} \subset R$ and $M$ is the maximal ideal, $M=M M^{-1}$ and $M^{-1}=\left(M:_{K} M\right)$. Thus, by Lemma $2.24, M^{-1} \cong M$. Therefore, by Corollary $2.14, M$ is a strongly G-projective $R$-module. 
If $M$ is an $R$-module, we let $\mu_{R}(M)$ denote the least number of elements required to generate $M$, and $\mu^{*}(R)=\sup \left\{\mu_{R}(I)\right\}$ where $I$ ranges over all finitely generated ideals of $R$.

Lemma 2.26 ([2, Proposition 1.4]). If $R$ is a Noetherian integral domain for which $\mu^{*}\left(R_{M}\right) \leqslant k$ for all maximal ideals $M$, then $\operatorname{dim}(R) \leqslant 1$ and $\mu^{*}(R) \leqslant$ $\max \{2, k\}$.

Theorem 2.27. Let $R$ be a Noetherian domain. Then $R$ is a Warfield domain if and only if, for any maximal ideal $M$ of $R, R_{M}$ is a 2-DVR.

Proof. First, we assume that $R$ is a Noetherian Warfield domain and $M$ is any maximal ideal of $R$. Then $R_{M}$ is also a Noetherian Warfield domain. So every ideal of $R_{M}$ can be generated by two elements. Thus, by Theorem $2.25, R_{M}$ is a 2-DVR.

Second, we assume that $R_{M}$ is a 2 -DVR for any maximal ideal $M$ of $R$. Then by Lemma $2.25, \mu^{*}\left(R_{M}\right) \leqslant 2$ for all maximal ideals $M$. Thus, by Lemma 2.26 , every ideal of $R$ can be generated by two elements. Therefore $R$ is a Warfield domain.

Acknowledgments. This work is partially supported by NSFC(No.11171240), and the doctoral foundation of Southwest University of Science and Technol$\operatorname{ogy}($ No.13zx7119).

\section{References}

[1] F. W. Anderson and K. R. Fuller, Rings and Categories of Modules, Grad. Texts in Math. 13, 2nd edition, Springer-Verlag, New York, 1992.

[2] H. Bass, Torsion free and projective modules, Trans. Amer. Math. Soc. 102 (1962), no. $2,319-327$.

[3] S. Bazzoni and L. Salce, Warfield domains, J. Algebra 185 (1996), no. 3, 836-868.

[4] D. Bennis, A note on Gorenstein global dimension of pullback rings, Int. Electron. J. Algebra 8 (2010), 30-44.

[5] D. Bennis and N. Mahdou, Strongly Gorenstein projective, injective, and flat modules, J. Pure Appl. Algebra 210 (2007), no. 2, 437-445.

[6] _ A generalization of strongly Gorenstein projective modules, J. Algebra Appl. 8 (2009), no. 2, 219-227.

[7] , Global Gorenstein dimensions, Proc. Amer. Math. Soc. 138 (2010), no. 2, 461465.

[8] I. S. Cohen, Commutative rings with restricted minimum condition, Duke Math. J. 17 (1950), 27-42.

[9] L. Fuchs and L. Salce, Modules over Non-Noetherian Domains, American Mathematical Society, Providence, RI., 2001.

[10] R. W. Gilmer, Multiplicative Ideal Theory, Marcel Dekker, New York, 1972.

[11] L. Gruson, Critères de platitude et de projectivité. Techniques de "platification" d'un module, Invent. Math. 13 (1971), 1-89.

[12] H. Holm, Gorenstein homological dimensions, J. Pure Appl. Algebra 189 (2004), no. 1-3, 167-193.

[13] K. Hu and F. Wang, Some results on Gorenstein Dedekind domains and their factor rings, Comm. Algebra 41 (2013), no. 1, 284-293.

[14] I. Kaplansky, Commutative Rings, Revised edition, Univ. Chicago Press, Chicago, 1974. 
[15] M. D. Larsen and P. J. McCarthy, Multiplicative Theory of Ideals, Academic Press, New York, 1971

[16] N. Mahdou and M. Tamekkante, On (strongly) Gorenstein (semi) hereditary rings, Arab. J. Sci. Eng. 36 (2011), no. 3, 431-440.

[17] E. Matlis, The two-generator problem for ideals, Michigan Math. J. 17 (1970), 257-265.

[18] H. Matsumura, Commutative Ring Theory, Cambridge University Press, New York, 1989.

[19] L. Salce, Warfield domains: module theory from linear algebra to commutative algebra through Abelian groups, Milan J. Math. 70 (2002), 163-185.

[20] F. Wang, Commutative Rings and Star Operation Theory (in Chinese), Science Press, Beijing, 2006.

Kui Hu

College of SCience

Southwest University of Science and Technology

Mianyang, 621010, P. R. China

E-mail address: hukui200418@163.com

FANGGUI WANG

College of Mathematics and Software Science

Sichuan Normal University

Chengdu, 610068, P. R. China

E-mail address: wangfg2004@163.com

LONGYU XU

College of Science

Southwest University of Science and Technology

Mianyang, 621010, P. R. China

E-mail address: xulongyu3@163.com

SONGQUAN ZHAO

College of Science

Southwest University of Science and Technology

Mianyang, 621010, P. R. China

E-mail address: z60198888@126.com 